\title{
Protein Kinase C Alpha Acting Through Phorbol-12- Myristate 13-Acetate Regulates Nephronectin Gene Expression Via c-Jun and c-Fos Transcription Factors
}

\author{
Mitsuhiro Kinoshita \\ Showa University \\ Atsushi Yamada ( $\nabla$ yamadaa@dent.showa-u.ac.jp ) \\ Showa University \\ Kiyohito Sasa \\ Showa University \\ Kaori lkezaki \\ Showa University \\ Tatsuo Shirota \\ Showa University \\ Ryutaro Kamijo \\ Showa University
}

\section{Research Article}

Keywords: Nephronectin (Npnt), phorbol 12-myristate 13-acetate (PMA), protein kinase C (PKC), siRNA

Posted Date: June 4th, 2021

DOI: https://doi.org/10.21203/rs.3.rs-579583/v1

License: (a) (i) This work is licensed under a Creative Commons Attribution 4.0 International License.

Read Full License 


\section{Abstract}

Nephronectin (Npnt) is an extracellular matrix protein and ligand of integrin $\alpha_{8} \beta_{1}$ known to promote differentiation of osteoblasts. A search for factors that regulate Npnt gene expression in osteoblasts revealed that phorbol 12-myristate 13-acetate (PMA), which activates protein kinase $C$ (PKC), had a strong effect to suppress that expression. Research was then conducted to elucidate the signaling pathway responsible for regulation of Npnt gene expression by PMA in osteoblasts. Treatment of MC3T3E1 cells with PMA suppressed cell differentiation and Npnt gene expression. Effects were noted at a low concentration of PMA, and were time- and dose-dependent. Furthermore, treatment with the PKC signal inhibitor Gö6983 inhibited down-regulation of Npnt expression, while transfection with small interfering RNA (siRNA) of PKCa, c-Jun, and c-Fos suppressed that down-regulation. The present results suggest regulation of Npnt gene expression via the PKCa and c-Jun/c-Fos pathway.

\section{Introduction}

The extracellular matrix surrounding cells is known to be involved in various biological functions, such as cell proliferation, differentiation, and apoptosis ${ }^{1-3}$. Several studies have suggested that the interaction of cells with the extracellular matrix is indispensable for histogenesis and maintenance of biological functions ${ }^{4,5}$. Nephronectin (Npnt) is an extracellular matrix protein considered to play critical roles in the development and function of various tissues ${ }^{6,7}$. Npnt gene expression is seen in calcification tissues, especially in osteoblasts, thus in order to investigate osteoblast functions, we have performed experiments to elucidate the pattern of $N p n t$ gene expression with several different reagents. In previous studies, we found that $1 \mathrm{a}, 25$-dihydroxyvitamin $\mathrm{D}_{3}$ and Wnt3a promoted Npnt gene expression ${ }^{8,9}$, whereas TGF- $\beta$, TNF- $\alpha$, IL-1 $\beta$, OSM, FGF-2, and inorganic phosphate suppressed that expression ${ }^{10-15}$. Those results suggest that $N p n t$ gene expression in osteoblasts is regulated via various factors. In a study conducted by Kahai S et al., an osteoblast-transfected Npnt gene expression vector was shown to promote differentiation ${ }^{16}$. Moreover, that differentiation was strongly promoted in cells in which the expressed region included EGF repeats. Also, in osteoblasts showing a high level of expression of mRNA $3^{\prime} U T R$ in the Npnt gene, the calcification nodule was highly promoted ${ }^{17}$.

PMA is a phorbol ester from the spurge family of plants and the main ingredient in croton oil, which causes strong carcinogenetic promotion activity. Protein kinase $C$ (PKC), which is activated by PMA, is a family of serine-threonine kinases that catalyze various biochemical reactions critical for the function of many cellular components, such as cell differentiation and proliferation ${ }^{18,19}$. The PKC family consists of 13 isoforms that can be divided into four subgroups based on their activated pattern ${ }^{20}$. Classical PKCs (cPKCs; $\alpha, \beta l, \beta I l, \gamma)$ require $\mathrm{Ca}^{2+} /$ diacylglycerol (DAG)/phosphatidylserine (PS), new PKCs (nPKCs; $\delta, \varepsilon, \eta$, $\theta)$ require DAG/PS, and atypical PKCs (aPKCs; $\lambda / l, \zeta)$ require PS, while so-called PKC-related kinases (PRKs; $1,2,3$ ), which are structurally distinct PKCs, require only PS for activation ${ }^{20}$. Activator protein 1 (AP-1) is a dimer consisting of the c-Jun, c-Fos, activating transcription factor (ATF), and musculoaponeurotic fibrosarcoma (MAF) families ${ }^{21}$. In most cells, the AP-1 morphology is 
predominantly a Jun/Fos heterodimer, which has a high affinity for binding to the PMA response component, thus is referred to as an AP-1 site ${ }^{22}$. It has also been reported that tumor promoters, such as PMA and epidermal growth factor, induce AP-1 activity ${ }^{23}$. The relationship of PKC and AP-1 has been investigated by analyses of their molecular mechanisms ${ }^{24,25}$.

In the present study, PMA was found to strongly inhibit Npnt gene expression through PKCa and the cJun/c-Fos pathway.

\section{Methods}

\section{Cell culture}

The osteoblast-like cell line MC3T3-E1 was maintained in MEMa with L-glutamine and phenol red medium (FujiFilm Wako Pure Chemical Industries, Ltd., Cat. No. 135-15175), supplemented with 10\% fetal bovine serum (FBS) (Biosera, Cat. No. FB-1285) and 1\% penicillin-streptomycin (Gibco, Cat.No. 15240$062)$ at $37^{\circ} \mathrm{C}$ in a $\mathrm{CO}_{2}$ incubator ( $5 \% \mathrm{CO}_{2}, 95 \%$ air). Osteoblast differentiation was induced by MEMa supplemented with $10 \%$ FBS and $100 \mathrm{ng} / \mathrm{ml}$ of BMP-2 (R\&D Systems, Cat. No.355-BEC-010) for three days.

\section{Reagents}

PMA (phorbol 12-myristate 13-acetate) was purchased from Adipo Gen Life Sciences, Inc. (Cat. No. AGCN2-0010-M001). BMP-2 human recombinant protein was purchased from R\&D Systems, Inc. (Cat. No.355-BEC-010) and Gö6983 from Cayman Chemical, Inc. (Cat. No.13311).

\section{Quantitative real-time PCR}

Total RNA was extracted from cells using TRIzol® Reagent (Life Technologies, Cat. No. 15596018), then cDNA was synthesized using ReverTra Ace ${ }^{\circledR}$ qPCR RT Master Mix (TOYOBO CO., LTD, Cat. No. FSQ-201). Quantitative real-time PCR was performed using Power Up ${ }^{\text {TM }}$ SYBR ${ }^{\text {TM }}$ Green Master Mix (Applied Biosystems, Cat. No. A25742) or THUNDERBIRD ${ }^{\circledR}$ Probe qPCR Mix (TOYOBO CO., LTD, Cat. No. QPS101). As another procedure, using TaqMan ${ }^{\mathrm{TM}}$ Fast Advanced Cells-to-CT ${ }^{\mathrm{TM}}$ Kit (Invitrogen) in accordance with the manufacturer's protocol, after cells were lysed cDNA was synthesized and then quantitative realtime PCR was performed. The TaqMan ${ }^{T M}$ IDs (Applied Biosystems) of the gene expression assay were as follows: Gapdh (Mm99999915_g1), Alp (Mm00475834_m1), and Osteocalcin (Mm03413826_mH). Following are the sequences of the specific PCR primers (Life Technologies):

Gapdh: 5'-AAATGGTGAAGGTCGGTGG-3' and 5'-TGAAGGGGTCGTTGATGG-3', Npnt. 5'CACGAGTAATTACGGTTGACAACAG - 3' and 5'-CTGCCGTGGAATGAACACAT-3'.

\section{Western blotting}

Cells were lysed with Sample Buffer Solution with Reducing Reagent (6x) for SDS-PAGE (NAKALAI TESQUE, Inc. Cat. No. 09499-14), then the lysates were subjected to SDS-PAGE. Following electrophoresis, 
proteins were transferred to PVDF membranes (Merck Millipore Ltd. Cat. No. IPVH00010). The membranes were treated with specific primary antibodies reacting to phospho-Marcks, Pkca, c-Jun, and cFos (Cell Signaling TCCHNOLOGY, Cat. No. 2741, 2056, 9165 and 4384, respectively), and actin (SIGMAALDRICH, Cat. No. A5060), followed by incubation with ECL ${ }^{\text {TM }}$ Anti-Rabbit IgG and treatment with a horseradish peroxidase linked whole antibody (GE Healthcare UK Limited Cat. No. NA934V). Immunoreactive bands were visualized using $\mathrm{ECL}^{\mathrm{TM}}$ Prime Western Blotting Detection Regents (GE Healthcare. Cat. No. RPN2232) and the intensity of chemi-luminescent bands was quantitated with Versa Doc 5000MP (Bio-Rad Laboratories, Inc.)

\section{ALP staining and activity}

Cells were fixed with $10 \%$ formalin in PBS, then ALP activity was visualized using a mixture of $0.1 \mathrm{mg} / \mathrm{ml}$ Naphthol As-Mx (SIGMA, Cat. No. N4875), 0.6 mg/ml phosphate, and Fast blue BB salt (SIGMA, Cat. No. F3378). For quantification of ALP activity, cells were disrupted by sonication in $50 \mathrm{mM}$ Tris- $\mathrm{HCl}$ containing $0.1 \%$ NP40 (Wako Pure Chemical Industries, Ltd., Cat. No.198596). ALP activity was determined following incubation with p-nitrophenylphosphate substrate (FujiFilm Wako Pure Chemical Industries, Ltd., Cat. No.149-02342).

\section{Knockdown of genes with RNA interference}

Cells were transfected with Stealth ${ }^{\mathrm{TM}}$ siRNAs for mouse $P k c a, c$-Jun siRNA, or a negative control (Invitrogen), or Silencer ${ }^{\mathrm{TM}}$ Select pre-designed siRNA for mouse c-Fos or a negative control (Ambion) using lipofectamine IMAX (Thermo Fisher) (Cat. No.13311), in accordance with the protocols of the manufacturers.

The respective oligos were as follows: Pkca. 5'-UCCAAAUGGGCUUUCGGAUCCUUAU-3' and 5'AUAAGGAUCCGAAAGCCCAUUUGGA-3', c-Jun: 5'-GAGAGCGGUGCCUACGGCUACAGUA-3' and 5'UACUGUAGCCGUAGGCACCGCUCUC-3', and c-Fos: 5'-CUACUUACACGUCUUCCUUtt-3' and 5'AAGGAAGACGUGUAAGUAGtg-3'.

\section{Statistical analysis}

Values are expressed as the mean \pm SD. A two-sided unpaired Student's test was used for statistical analysis. Statistical differences were considered to be significant when the $p$ value was $<0.05$.

\section{Results}

PMA suppresses BMP-2 induced osteoblast differentiation in MC3T3-E1 cells.

To investigate the effect of PMA on osteoblastic differentiation, MC3T3-E1 cells were cultured with BMP$2(100 \mathrm{ng} / \mathrm{ml})$ in the absence or presence of PMA $(5 \mathrm{nM})$ for three days. ALP activity in cells cultured with BMP-2 was shown to be increased, whereas it was significantly suppressed when cells were cultured in the combination of BMP-2 and PMA (Fig. 1Aa, b). At the same time, the gene expressions of Alp and Osteocalcin, differentiation markers of osteoblasts, were investigated. Both Alp and Osteocalcin gene 
expressions induced by BMP-2 were suppressed by PMA. These results showed that PMA suppressed BMP-2 induced osteoblast differentiation (Fig. 1B) ${ }^{26}$.

Npnt gene expression is suppressed by PMA in dose and time-dependent manner.

PMA, a phorbol ester, is known to activate the PKC signaling pathway. To determine whether PMA activated the PKC signaling pathway in MC3T3-E1 cells, Marcks phosphorylation was examined, as previous studies have reported that it was phosphorylated by PKC activation 27,28. Marcks was remarkably phosphorylated by PMA (Fig. 2A). The effect of PMA on Npnt gene expression was also examined and the results showed that expression to be significantly down-regulated by PMA (Fig. 2B). Next, the effects of PMA on dose- and time-dependent Npnt gene expression were investigated. That expression was significantly decreased by PMA at $3.2 \mathrm{nM}$ and reached a plateau at $32 \mathrm{nM}$ (Fig. 2C), while it was also significantly decreased by $10 \mathrm{nM}$ of PMA at 12 hours and then reached a plateau at 24 hours (Fig. 2D). These results suggest that Npnt gene expression is suppressed by PMA in a dose and timedependent manner.

PKCa is involved in down-regulation of Npnt gene expression by PMA.

To verify whether down-regulation of Npnt gene expression by PMA is involved in the PKC signaling pathway, MC3T3-E1 cells were pretreated with Gö6983, known as a broad-spectrum PKC inhibitor, before PMA stimulation. Phosphorylation of Marks by PMA did not occur following pretreatment with Gö6983 (Fig. 3A), while down-regulation of Npnt gene expression by PMA was inhibited by Gö6983 (Fig. 3B). These results suggest that Npnt gene expression is involved in the PKC signaling pathway.

It has been reported that PKCa is highly expressed in MC3T3-E1 cells ${ }^{29}$. To verify its involvement in down-regulation of Npnt gene expression, MC3T3-E1 cells were pretreated with or without $P k c a$ siRNA, and thereafter with PMA alone or in combination. When Pkca siRNA decreased the cellular protein level of Pkca (Fig. 3C), down-regulation of Npnt gene expression by PMA was inhibited (Fig. 3D). These results indicate that PKCa is involved in down-regulation of Npnt gene expression by PMA.

Both of c-Jun and c-Fos are involved in down-regulation of Npnt gene expression.

It has been reported that regulation of gene expression by PMA is involved in activation of PKCa and thereafter of AP-1 ${ }^{30}$. To investigate the involvement of c-Jun and c-Fos as transcription factors, which compose AP-1, on down-regulation of Npnt gene expression, MC3T3-E1 cells were pretreated with or without c-Jun, c-Fos siRNA, and then treated with PMA alone or in combination. When c-Jun siRNA decreased the cellular protein level of c-Jun (Fig. 4A), down-regulation of Npnt gene expression by PMA was inhibited (Fig. 4B), and when c-Fos siRNA decreased the level of c-Fos mRNA (Fig. 4C), downregulation of Npnt gene expression by PMA was inhibited (Fig. 4D). These results demonstrated that the transcription factors c-Jun and c-Fos are involved in down-regulation of Npnt gene expression by PMA.

\section{Discussion}


The present findings indicate that PMA, known to suppress osteoblast differentiation, downregulates Npnt gene expression. That downregulation was shown to be mediated via PKCa, and further via c-Jun and c-Fos, which are transcription factors in PKC signaling. Nakura A. et al., demonstrated that knockdown of $P K C a$ gene expression promoted osteoblast differentiation and their results also suggest that PKCa suppresses osteoblast differentiation ${ }^{31}$. Furthermore, Galea, G.L. et al. reported that PKCa knockout mice, which show a phenotype similar to human Gaucher disease, had bone formation into the medullary space of the femur. Moreover, osteoblasts derived from those mice showed elevated osteoblast differentiation markers, such as Runx2, Osterix, Col1A1, and Osteocalcin ${ }^{32}$. Together, these results suggest that PKCa negatively regulates bone formation. Additionally, the present results indicate that PKCa negatively regulates promotion of osteoblast differentiation, with one of the causes considered to be a decrease in Npnt gene expression due to PKCa, though further studies are required to confirm that association.

c-Jun, c-Fos siRNA decreased the level of c-Jun, c-Fos mRNA, which resulted in partial recovery of downregulation of Npnt gene expression by PMA. This suggests the presence of another pathway in addition to the c-Jun and c-Fos pathways for suppressing Npnt gene expression by PMA. Bedini A. et al., reported that PMA treatment suppressed $h M O R$ gene expression in SH-SY5Y cells, the neuroblastoma cell line. In addition, in the present study, suppression of expression of REST (repressor element 1 silencing transcription factor), a transcription factor known to be involved in regulation of gene expression in differentiated and post-differentiated neurons, inhibited PMA-induced hMOR gene downregulation. The hMOR promoter has been shown to have a REST binding region ${ }^{33}$. Furthermore, Kuan C.S. et al., reported that PMA treatment suppressed $c k \beta$ gene expression in MCF-7 cells, while it also suppressed the promoter activity of the $c k \beta$ gene ${ }^{34}$. That study also noted that the promoter region of the $c k \beta$ gene has binding sites for the transcription factors GATA and Ets, and mutations in those binding sites inhibited suppression of the promoter activity of the $c k \beta$ gene by PMA. It is considered that these transcription factors may be involved in suppression of Npnt gene expression by PMA, though additional research is needed to verify their relationship.

In conclusion, we found that PKCa suppresses Npnt gene expression via c-Jun and c-Fos transcription factors (Fig. 5).

\section{Declarations}

\section{Authors' contributions}

$\mathrm{MK}, \mathrm{AY}, \mathrm{TS}$, and RK designed the experiments. MK, KI, and KS performed corresponding experiments. MK, $A Y$, and RK wrote the paper.

\section{Acknowledgements}


We greatly appreciate Dr. S. Ohba, Dr. H. Hojo, and Dr. N. Morimura for their fruitful discussions. This work was supported in part by grants from JSPS Kakenhi (20K21687). The authors have no financial conflicts of interest to declare.

\section{References}

1. Makino, H., Sugiyama, H. \& Kashihara, N. Apoptosis and extracellular matrix-cell interactions in kidney disease. Kidney Int Supp/77, S67-75 (2000).

2. Schlie-Wolter, S., Ngezahayo, A. \& Chichkov, B. N. The selective role of ECM components on cell adhesion, morphology, proliferation and communication in vitro. Exp Cell Res 319, 1553-1561, doi:10.1016/j.yexcr.2013.03.016 (2013).

3. Lin, C. Q. \& Bissell, M. J. Multi-faceted regulation of cell differentiation by extracellular matrix. Fasebj 7, 737-743, doi:10.1096/fasebj.7.9.8330681 (1993).

4. Ashkenas, J., Muschler, J. \& Bissell, M. J. The extracellular matrix in epithelial biology: shared molecules and common themes in distant phyla. Dev Biol 180, 433-444, doi:10.1006/dbio.1996.0317 (1996).

5. Karsdal, M. A. et al. Novel insights into the function and dynamics of extracellular matrix in liver fibrosis. Am J Physiol Gastrointest Liver Physio/ 308, G807-830, doi:10.1152/ajpgi.00447.2014 (2015).

6. Morimura, N. et al. Molecular cloning of POEM: a novel adhesion molecule that interacts with alpha8beta1 integrin. J Biol Chem 276, 42172-42181, doi:10.1074/jbc.M103216200 (2001).

7. Brandenberger, R. et al. Identification and characterization of a novel extracellular matrix protein nephronectin that is associated with integrin alpha8beta1 in the embryonic kidney. J Cell Bio/ 154, 447-458, doi:10.1083/jcb.200103069 (2001).

8. Hiranuma, K. et al. Expression of nephronectin is enhanced by 1a,25-dihydroxyvitamin D3. FEBS Open Bio 6, 914-918, doi:10.1002/2211-5463.12085 (2016).

9. Ikehata, M. et al. Wnt/ $\beta$-catenin signaling activates nephronectin expression in osteoblasts. Biochem Biophys Res Commun 484, 231-234, doi:10.1016/j.bbrc.2017.01.053 (2017).

10. Miyazono, A. et al. TGF-beta suppresses POEM expression through ERK1/2 and JNK in osteoblasts. FEBS Lett 581, 5321-5326, doi:10.1016/j.febslet.2007.10.021 (2007).

11. Tsukasaki, M. et al. Expression of POEM, a positive regulator of osteoblast differentiation, is suppressed by TNF-a. Biochem Biophys Res Commun 410, 766-770, doi:10.1016/j.bbrc.2011.06.048 (2011).

12. lezumi, Y. et al. IL-1 $\beta$ suppresses nephronectin expression in osteoblasts via ERK1/2 and JNK. Biochem Biophys Res Commun 493, 773-775, doi:10.1016/j.bbrc.2017.08.104 (2017).

13. Kurosawa, T. et al. Expression of nephronectin is inhibited by oncostatin $M$ via both JAK/STAT and MAPK pathways. FEBS Open Bio 5, 303-307, doi:10.1016/j.fob.2015.04.001 (2015). 
14. Kato, T. et al. FGF-2 suppresses expression of nephronectin via JNK and PI3K pathways. FEBS Open Bio 8, 836-842, doi:10.1002/2211-5463.12421 (2018).

15. Kato, T. et al. Nephronectin Expression is Inhibited by Inorganic Phosphate in Osteoblasts. Calcif Tissue Int 104, 201-206, doi:10.1007/s00223-018-0484-3 (2019).

16. Kahai, S., Lee, S. C., Seth, A. \& Yang, B. B. Nephronectin promotes osteoblast differentiation via the epidermal growth factor-like repeats. FEBS Lett 584, 233-238, doi:10.1016/j.febslet.2009.11.077 (2010).

17. Kahai, S. et al. MicroRNA miR-378 regulates nephronectin expression modulating osteoblast differentiation by targeting GalNT-7. PLoS One 4, e7535, doi:10.1371/journal.pone.0007535 (2009).

18. Jeong, H. M. et al. PKC signaling inhibits osteogenic differentiation through the regulation of Msx2 function. Biochim Biophys Acta 1823, 1225-1232, doi:10.1016/j.bbamcr.2012.05.018 (2012).

19. Song, J. K. et al. Effect of phorbol 12-myristate 13-acetate on the differentiation of adipose-derived stromal cells from different subcutaneous adipose tissue depots. Korean J Physiol Pharmacol 18, 289-296, doi:10.4196/kjpp.2014.18.4.289 (2014).

20. da Rocha, A. B., Mans, D. R., Regner, A. \& Schwartsmann, G. Targeting protein kinase C: new therapeutic opportunities against high-grade malignant gliomas? Oncologist 7, 17-33, doi:10.1634/theoncologist.7-1-17 (2002).

21. Eferl, R. \& Wagner, E. F. AP-1: a double-edged sword in tumorigenesis. Nat Rev Cancer 3, 859-868, doi:10.1038/nrc1209 (2003).

22. Buttice, G., Quinones, S. \& Kurkinen, M. The AP-1 site is required for basal expression but is not necessary for TPA-response of the human stromelysin gene. Nucleic Acids Res 19, 3723-3731, doi:10.1093/nar/19.13.3723 (1991).

23. Young, M. R. et al. Transactivation of Fra-1 and consequent activation of AP-1 occur extracellular signal-regulated kinase dependently. Mol Cell Biol 22, 587-598, doi:10.1128/mcb.22.2.587-598.2002 (2002).

24. Wang, Y., Schattenberg, J. M., Rigoli, R. M., Storz, P. \& Czaja, M. J. Hepatocyte resistance to oxidative stress is dependent on protein kinase C-mediated down-regulation of c-Jun/AP-1. J Biol Chem 279, 31089-31097, doi:10.1074/jbc.M404170200 (2004).

25. Blumenthal, E. J., Copestake, C., Hite, G. \& Hoversland, R. C. Pregnancy-associated, lymphocytederived suppressor factor inhibits protein kinase $C$ activity. J Reprod Immuno/ 24, 97-109, doi:10.1016/0165-0378(93)90013-8 (1993).

26. Boguslawski, G. et al. Activation of osteocalcin transcription involves interaction of protein kinase Aand protein kinase C-dependent pathways. J Biol Chem 275, 999-1006, doi:10.1074/jbc.275.2.999 (2000).

27. Eliyahu, E., Shtraizent, N., Tsaadon, A. \& Shalgi, R. Association between myristoylated alanin-rich C kinase substrate (MARCKS) translocation and cortical granule exocytosis in rat eggs. Reproduction 131, 221-231, doi:10.1530/rep.1.00794 (2006). 
28. Luo, B., Prescott, S. M. \& Topham, M. K. Association of diacylglycerol kinase zeta with protein kinase C alpha: spatial regulation of diacylglycerol signaling. J Cell Bio/ 160, 929-937, doi:10.1083/jcb.200208120 (2003).

29. Lampasso, J. D., Chen, W. \& Marzec, N. The expression profile of PKC isoforms during MC3T3-E1 differentiation. Int $J$ Mol Med 17, 1125-1131 (2006).

30. Palazzo, E. et al. A novel DLX3-PKC integrated signaling network drives keratinocyte differentiation. Cell Death Differ 24, 717-730, doi:10.1038/cdd.2017.5 (2017).

31. Nakura, A., Higuchi, C., Yoshida, K. \& Yoshikawa, H. PKCa suppresses osteoblastic differentiation. Bone 48, 476-484, doi:10.1016/j.bone.2010.09.238 (2011).

32. Galea, G. L. et al. Protein kinase $\mathrm{Ca}(\mathrm{PKCa})$ regulates bone architecture and osteoblast activity. J Biol Chem 289, 25509-25522, doi:10.1074/jbc.M114.580365 (2014).

33. Bedini, A., Baiula, M., Carbonari, G. \& Spampinato, S. Transcription factor REST negatively influences the protein kinase $\mathrm{C}$-dependent up-regulation of human mu-opioid receptor gene transcription. Neurochem Int 56, 308-317, doi:10.1016/j.neuint.2009.10.014 (2010).

34. Kuan, C. S., Yee, Y. H., See Too, W. C. \& Few, L. L. Ets and GATA transcription factors play a critical role in PMA-mediated repression of the $\mathrm{ck} \beta$ promoter via the protein kinase $\mathrm{C}$ signaling pathway. PLoS One 9, e113485, doi:10.1371/journal.pone.0113485 (2014).

\section{Figures}


A

a)

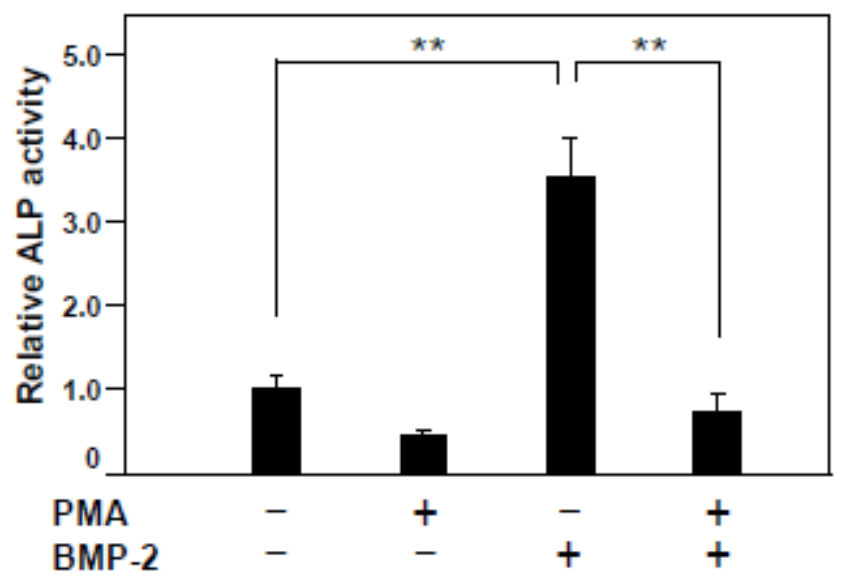

B

a)

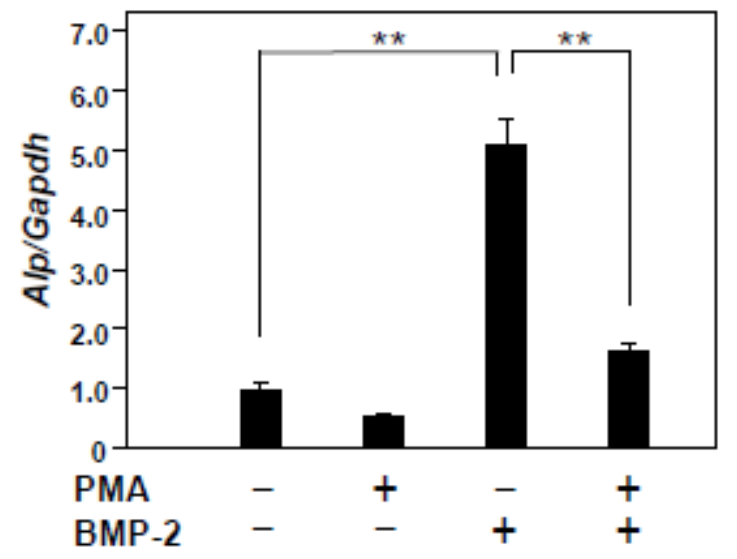

b)

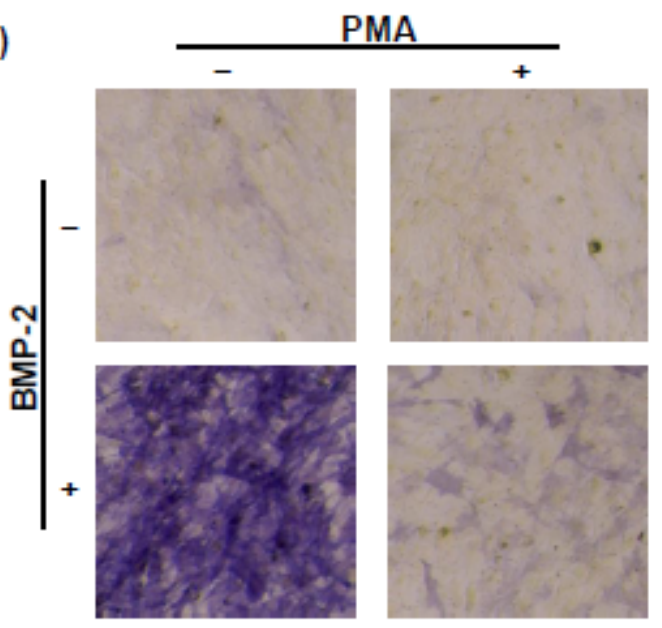

b)

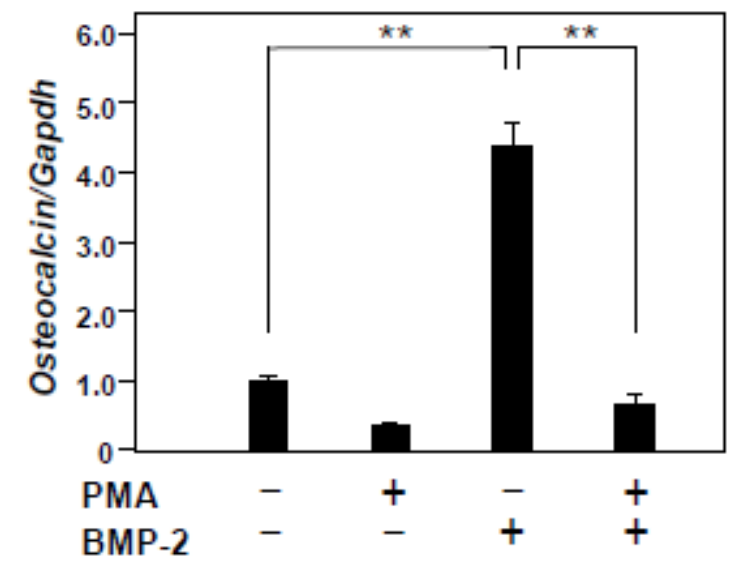

\section{Figure 1}

Effects of PMA on BMP-2 induced osteoblast differentiation in MT3T3-E1 cells. (A) (a) MC3T3-E1 cells were treated with or without BMP-2 $(100 \mathrm{ng} / \mathrm{ml})$ in the presence or absence of PMA $(5 \mathrm{nM})$ for three days. For quantification of ALP activity, cells were disrupted by sonication in $50 \mathrm{mM}$ Tris- $\mathrm{HCl}$ containing $0.1 \%$ NP40. ALP activity was determined following incubation with the substrate p-nitrophenylphosphate and using absorbance at $405 \mathrm{~nm}$. (b) For ALP staining, cells were fixed using 10\% formalin in PBS and then ALP activity was visualized using a mixture of $0.1 \mathrm{mg} / \mathrm{ml}$ Naphthol As-Mx, $0.6 \mathrm{mg} / \mathrm{ml}$ phosphate, and Fast blue BB salt. (B) Total cellular RNA was extracted, then mRNA levels of Alp, Osteocalcin, and Gapdh were examined using quantitative real-time PCR analysis. Results are shown as the mean \pm SD of three samples. ${ }^{* * P}<0.01$, Student's t test. 
A

PMA

$+$

p-Marcks

Actin

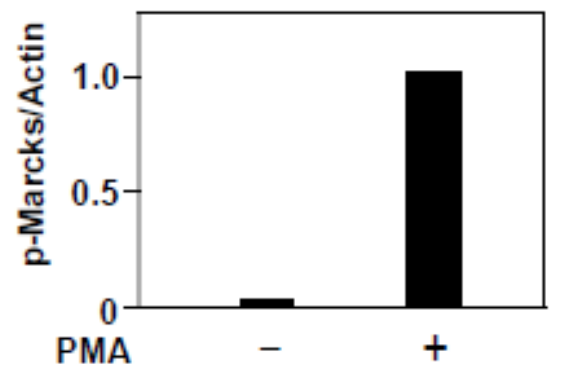

C

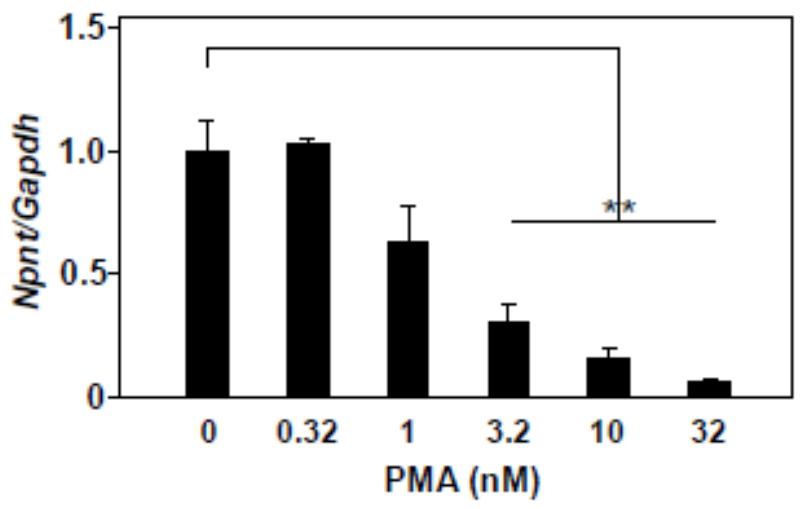

B

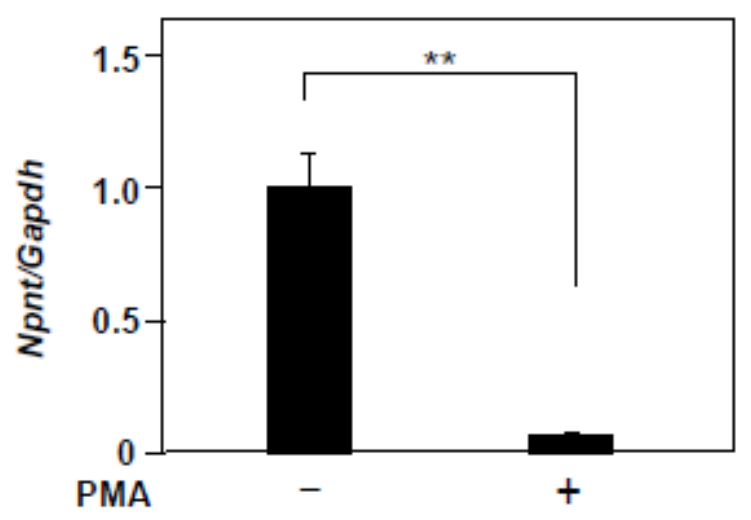

D

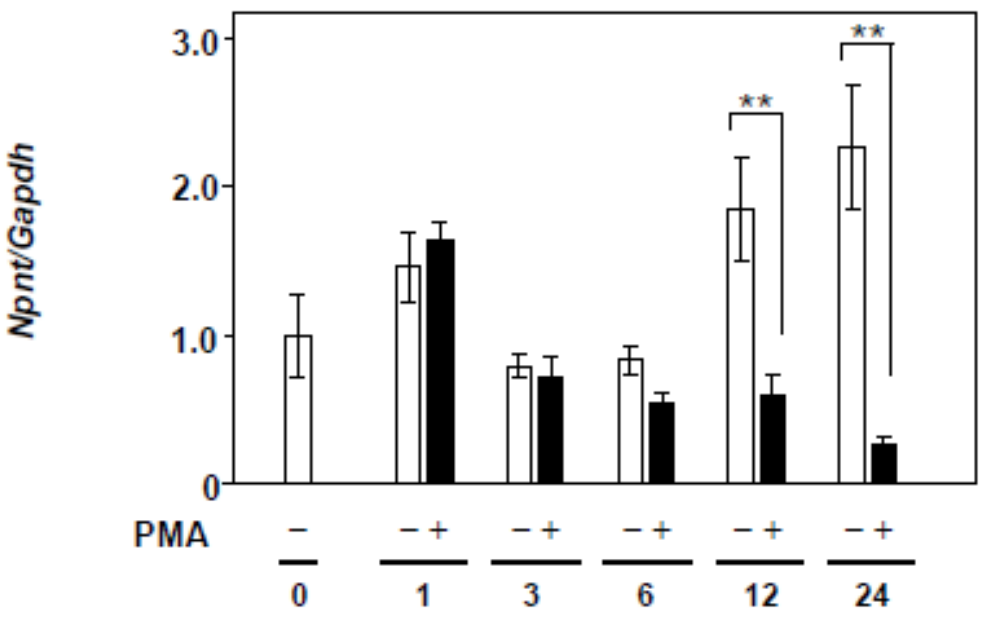

Culture period (h)

Figure 2

Effects of PMA on Npnt gene expression. (A) MC3T3-E1 cells were starved for 16 hours in serum-free medium. Cells were treated with or without PMA (100 nM) for five minutes, then proteins were extracted and subjected to western blotting to detect phosphorylation of Marks (p-Marks) and actin. (B) MC3T3-E1 cells were treated with PMA (10 nM) for 24 hours. Total cellular RNA was extracted, and mRNA levels of Npnt and Gapdh were examined using quantitative real-time PCR analysis. (C) Dose-dependent effects of PMA on Npnt expression. MC3T3-E1 cells were treated with PMA $(0,0.32,1,3.2,10$, or 32 nM) for 24 hours and then examined using quantitative real-time PCR analysis. (D) Time course analysis of PMA effects on Npnt gene expression. MC3T3-E1 cells were treated with PMA (10 nM) for 1, 3, 6, 12, or 24 hours and then examined using quantitative real-time PCR analysis. Results are shown as the mean \pm SD of 3 samples. ${ }^{*} \mathrm{P}<0.01$, Student's t-test as compared to the level with $0 \mathrm{nM}$ of PMA. 
A
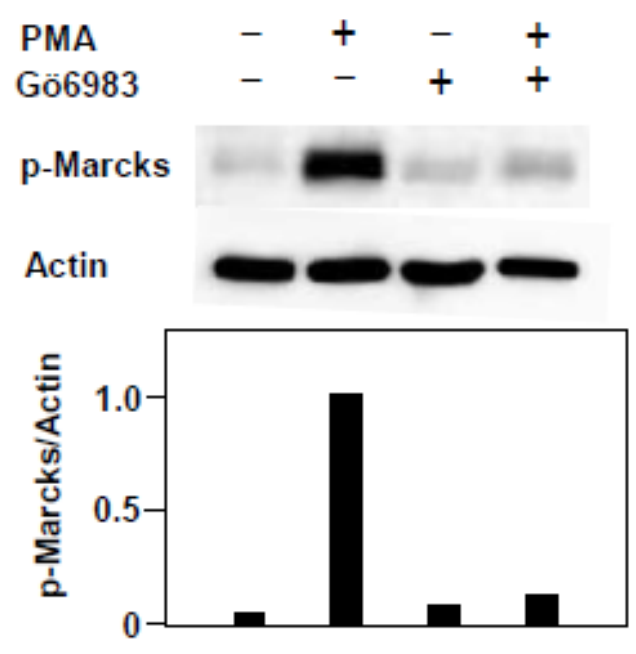

$\mathrm{C}$

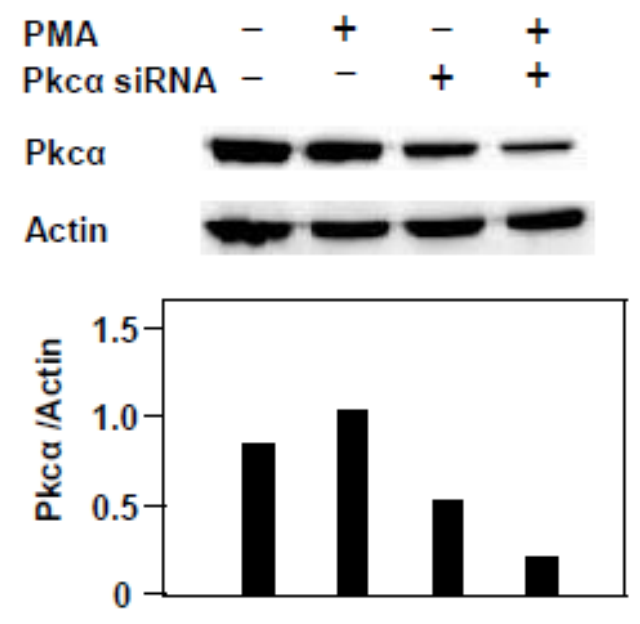

B

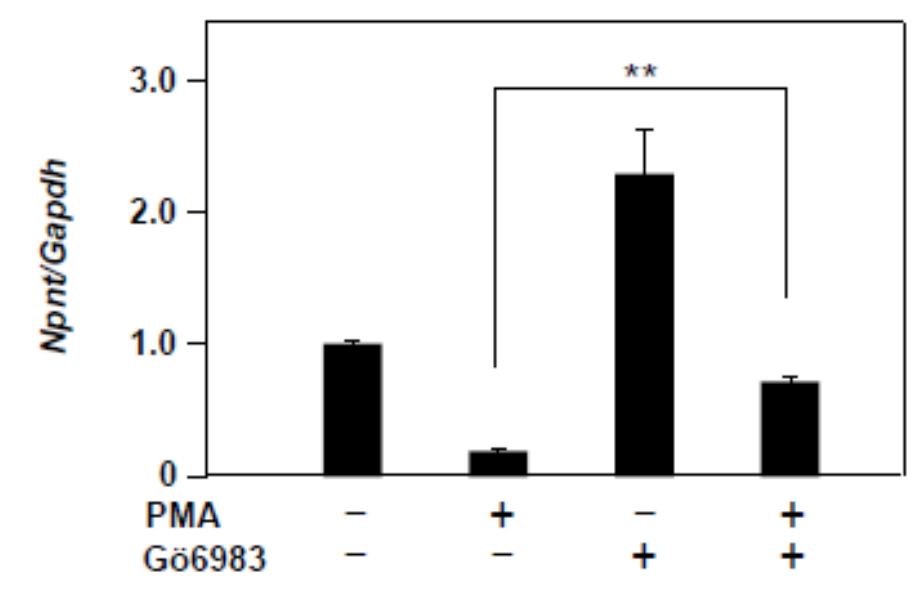

D

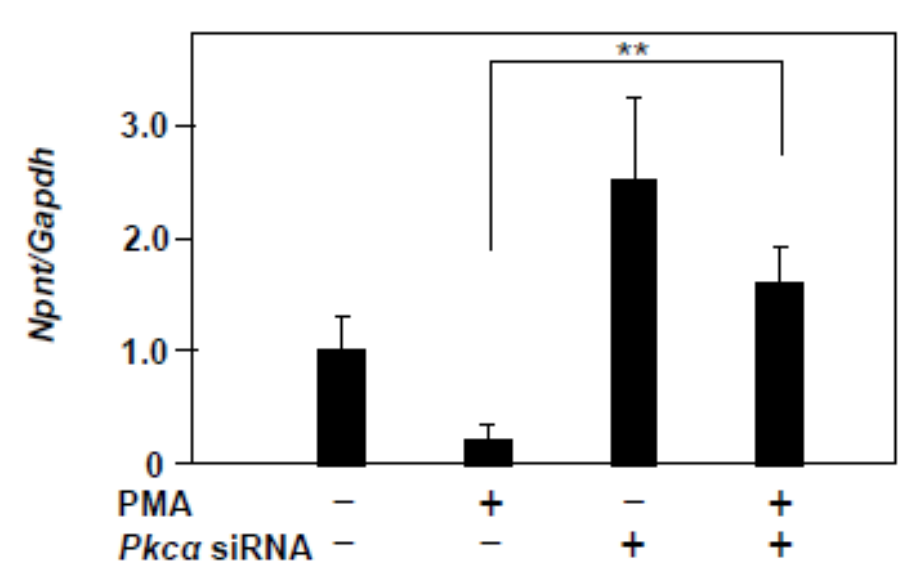

\section{Figure 3}

PKC signaling, especially PKCa, is involved in Npnt gene down-regulation by PMA. (A) MC3T3-E1 cells were starved for 16 hours in serum-free medium. Next, they were pretreated with or without Gö6983 (500 $\mathrm{nM})$ for one hour, and then with PMA ( $5 \mathrm{nM})$ alone or in combination for five minutes. Proteins were extracted and subjected to western blotting to detect phosphorylation of Marks (p-Marks) and actin. (B) MC3T3-E1 cells were pretreated with or without Gö6983 (500 nM) for one hour, and then treated with PMA ( $5 \mathrm{nM}$ ) alone or in combination for 24 hours. Total cellular RNA was extracted, and mRNAs for Npnt and Gapdh were examined using real-time PCR analysis. (C) MC3T3-E1 cells were pretreated with or without Pkca siRNA (20 nM) for 24 hours, and then treated with PMA (10 nM) alone or in combination for 24 hours. Proteins were extracted and subjected to western blotting to detect Pkca and actin. (D) Total cellular RNA was extracted, and mRNAs for Npnt and Gapdh were examined using real-time PCR analysis. Results are shown as the mean \pm SD of three samples. ${ }^{*} P<0.01$, Student's t-test, as compared to presence or absence of PMA, Gö6983, and Pkca siRNA. 
A
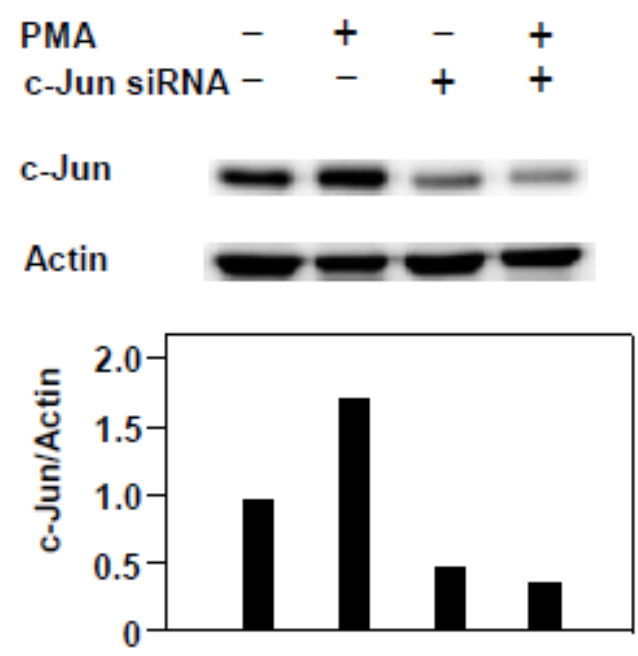

C
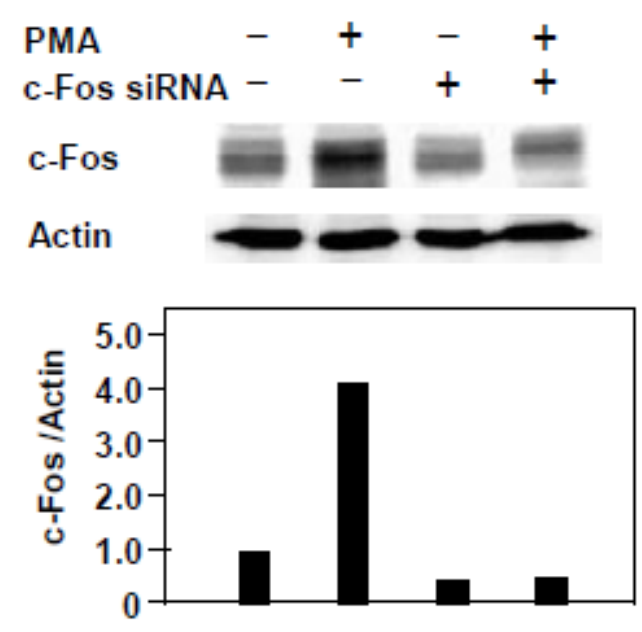

B

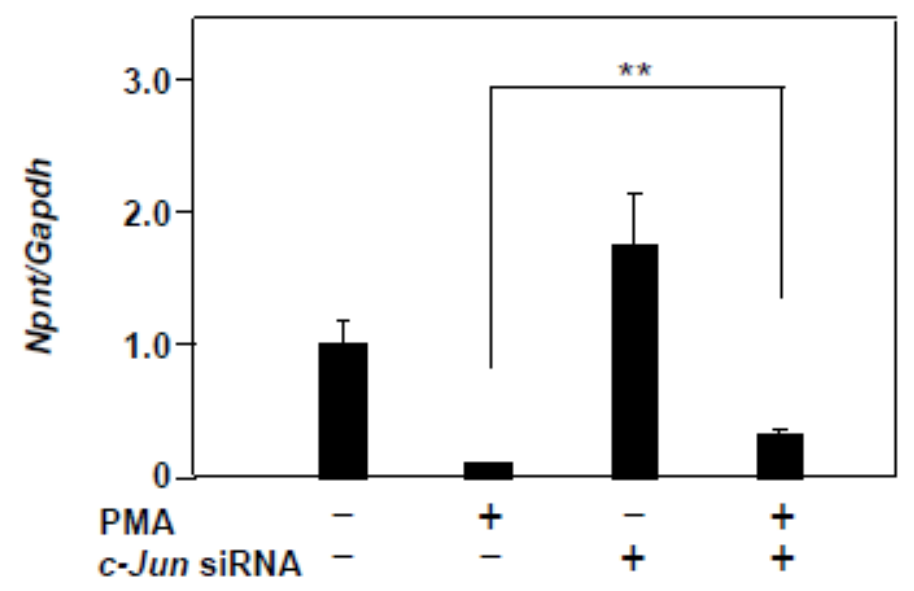

D

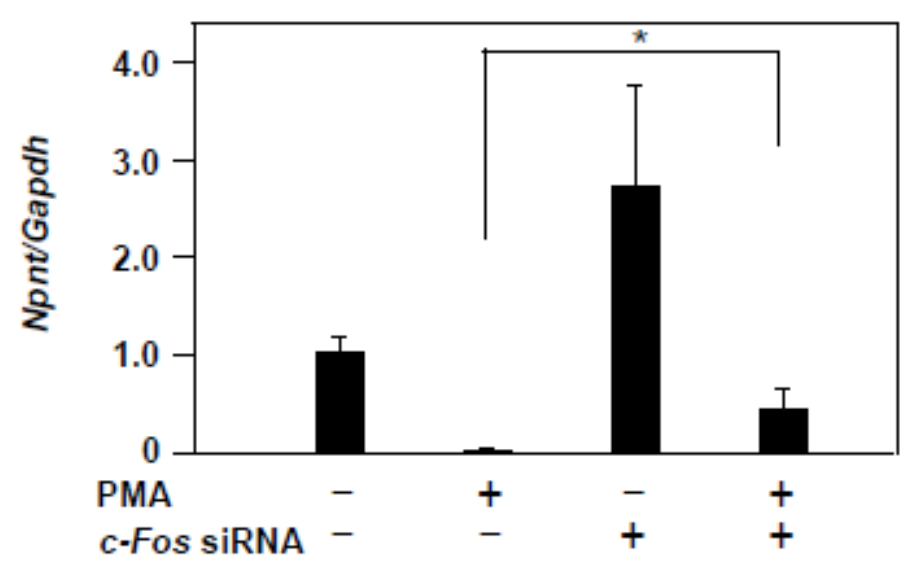

\section{Figure 4}

Npnt gene down-regulation by PMA regulated via c-Jun and c-Fos transcription factors. MC3T3-E1 cells were pretreated with or without c-Jun siRNA $(20 \mathrm{nM})$ or c-Fos siRNA $(20 \mathrm{nM})$ for 24 hours, and then treated with PMA (100 nM) alone or in combination for 24 hours with c-Jun or for three hours with c-Fos. (A) Proteins were extracted and subjected to western blotting to detect c-Jun and actin. (B) Total cellular RNA was extracted, and mRNAs for Npnt and Gapdh were examined using real-time PCR analysis. (C) Proteins were extracted using the same procedures shown in (A) and (B), and subjected to western blotting to detect c-Fos and Actin. (D) Total cellular RNA was extracted, and mRNAs for Npnt and Gapdh were examined using real-time PCR analysis. Results are shown as the mean \pm SD of three samples. ${ }^{*}$ $<0.05$, ${ }^{\star *} \mathrm{P}<0.01$, Student's t-test, as compared to presence or absence of PMA, c-Jun siRNA, and c-Fos SiRNA. 


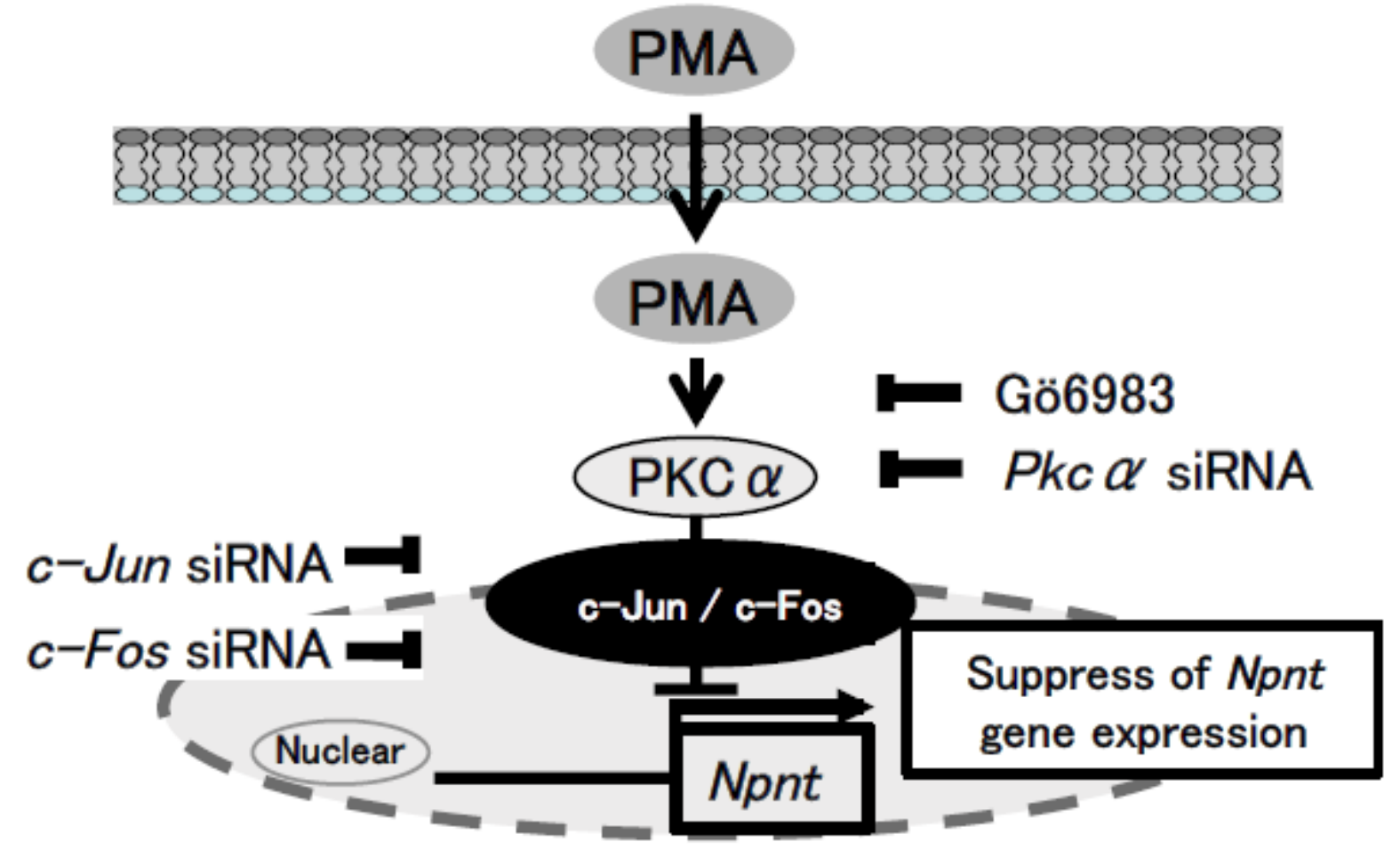

Figure 5

Model of down-regulation of Npnt gene expression by PMA. Activation of PKC signaling by PMA, Npnt gene expression was suppressed via the transcription factors c-Jun and c-Fos. 